\title{
Learning-Based Modeling of Endovascular Navigation for Collaborative Robotic Catheterization
}

\author{
Hedyeh Rafii-Tari ${ }^{1}$, Jindong Liu ${ }^{1}$, Su-Lin Lee ${ }^{1}$, \\ Colin Bicknell ${ }^{2}$, and Guang-Zhong Yang ${ }^{1}$ \\ 1 The Hamlyn Centre for Robotic Surgery \\ 2 Academic Division of Surgery \\ Imperial College London, UK \\ \{h.rafii-tari11,j.liu,g.z.yang\}@imperial.ac.uk
}

\begin{abstract}
Despite rapid growth of robot assisted catheterization in recent years, most current platforms are based on master-slave designs with limited operator-robot collaborative control and automation. Under this setup, information concerning subject specific behavior and context-driven manoeuvre is not re-utilized for subsequent intervention. For endovascular catheterization, the robot itself is designed with little consideration of underlying skills and associated motion patterns. This paper proposes a learning-based approach for generating optimum motion trajectories from multiple demonstrations of a catheterization task such that it can be used for automating catheter motion within a collaborative setting. Motion models are generated from experienced manipulation of a catheterization procedure and replicated using a robotic catheter driver to assist inexperienced operators. Catheter tip motions of the automated approach are compared against the manual training sets for validating the proposed framework. The results show significant improvements in the quality of catheterization, which facilitate the design of hands-on collaborative robots that make full use of the natural skills of the operators.
\end{abstract}

\section{Introduction}

Remote-controlled steerable catheter navigation systems are enjoying growing interests in recent years, particularly for endovascular surgery. Advantages of these robots include increased stability, elimination of tremor, added operator comfort and reduced exposure to ionizing radiation as compared to manual catheterization [1. Robotic systems can offer possibilities for automating whole or parts of the procedures that are more difficult to perform, while reducing the cognitive workload of the operator. Most current designs, however, do not take into consideration the skilled motion patterns and operator-tool interactions used in practice. Using multiple demonstrations of catheterization tasks to capture these underlying patterns, and applying them towards semi-autonomous robotic 
catheter insertion within a collaborative framework can have a significant impact on improving the performance of catheterization tasks.

One of the main commercial robotic platforms for endovascular intervention, including aneurysm repair and balloon angioplasty, is the Magellan System (Hansen Medical, CA, USA) 2]. Extensive research has also been conducted for the development of robotic master/slave solutions for remote control of standard catheters 3. Control of the catheter in most of these cases, however, is achieved by using a multi-degree-of-freedom (DoF) haptic interface that potentially alters the natural behavior and motion patterns of experienced operators. This has called for the development of robotic systems whose master interface replicates the motions used during standard catheterization practice [4.

In endovascular intervention, catheters are advanced over guidewires to the target site using a combination of insertion, retraction, and torque at the proximal end while 2D fluoroscopy is typically used for guidance. Studies have shown distinct differences between expert and novice operators in the force/torque patterns exerted on the catheter and their relation to catheter motion [5]. The steep learning curves associated with endovascular intervention 6] further motivate the understanding of underlying behavioural patterns and the development of ergonomically sound robotic platforms that can replicate the natural manipulation skills learned from standard catheterization tasks. This can have a significant impact on reducing complications that are caused as a result of interactions between the catheter and the arterial wall, including dissection, thrombosis, embolization and perforation [7. The incorporation of motion and contact force sensing can further enhance the safety and consistency of the procedures 8$]$.

Thus far, all the motion and sensing information captured during endovascular intervention are mainly used for the purpose of skills assessment. It is envisaged that such information can also be used for automating certain steps of the surgical task under the general learning-from-demonstration framework in robotics [9]. Learned models from multiple demonstrations of tele-robotic surgical motions have already been implemented in other areas of minimally invasive surgery [10, and used for shared control between operator and robot [1].

This paper proposes a learning-based approach for robot-assisted endovascular catheterization. A proximal position sensor is used to measure the axial/rotational motion of the catheter and a learning-from-demonstration framework is developed to encode the motion patterns and generate smooth trajectories that capture the main features of the training data. A hands-on robot for real-time assistance within a collaborative setting has been developed, through which guidewire manipulation is controlled manually by the operator while catheter motion is automated by the robot. Since successful catheterization relies on in-sync manipulation of both catheter and guidewire, this collaborative scheme utilizes operator skill and ensures safety through fine-tuning the motions if needed. Different motion trajectories are generated from demonstrations of expert and intermediate-level operators and validated using the robot across multiple inexperienced operators. The performance of the system is evaluated by comparing catheter motion quality with and without the use of the robot 
incorporating adaptive learning. Issues concerning inter/intra-operator variability for learning from multiple demonstrations across different operators have also been addressed by dividing the procedure into phases. The method proposed provides an effective means of learning underlying skills and extracting characteristic features of endovascular navigation to enable semi-automated robotic catheterization, with generic applicability to other surgical robots.

\section{Materials and Methods}

\subsection{Hardware and Experimental Setup}

To collect training data across multiple demonstrations, a motion sensor is designed to measure the two DoF linear and rotational motion of the catheter applied by the operator at the proximal end as shown in Fig. 1, by using two independent sensors consisting of contact less magnetic rotary encoders (4096 counts/rev resolution, AS5145H Austriamicrosystems). Axial position measurement was obtained by passing the catheter through customized micro-rollers, with the magnet of the encoder directly coupled to the shaft of one of the rollers. Radial motion is measured using a gear-based coupling between the axial component and the base. The sensor measurements were read into Labview using an acquisition card (NI-USB6009, National Instruments Corp.). Axial measurement was calculated from the sensor readings using the circumference of the roller, resulting in a motion sensitivity of $0.0054 \mathrm{~mm} /$ count in the axial direction and a sensitivity of 0.0879 degrees/count in the radial direction.

For the collection of motion data and validation of learned trajectories, a silicone-based, anthropomorphic phantom (Elastrat Sarl, Switzerland) of a type I aortic arch was used for this study. The selected procedure was cannulation of the innominate artery in the model. Using the axial and rotational motion data obtained from the proximal sensor, motion models were learned from multiple demonstrations of the task by an expert operator $(\mathrm{n}=6$, more than 100 endovascular procedures) as well as demonstrations by two intermediate level operators ( $\mathrm{n}=8$, less than 10 simulator/endovascular procedures) and separate trajectories were extracted for each group. A camera was mounted above the phantom and the $2 \mathrm{D}$ projected image was used by operators for navigation. A $5 \mathrm{~F}$ shaped catheter and a guidewire were used in this study. For validation purposes, information regarding the catheter tip motion was also obtained using a six DoF electromagnetic position sensor (Aurora, NDI) attached to the catheter tip. The experimental setup is shown in Fig. 1.

\subsection{Catheter Motion Modeling}

The axial and rotational signals obtained from the proximal position sensors were first smoothed using a moving average filter ( $\operatorname{span}=3$ ). To address inter/intraoperator variability and ensure generation of smooth trajectories that translate to seamless robot execution in stages, the obtained motion data were divided 

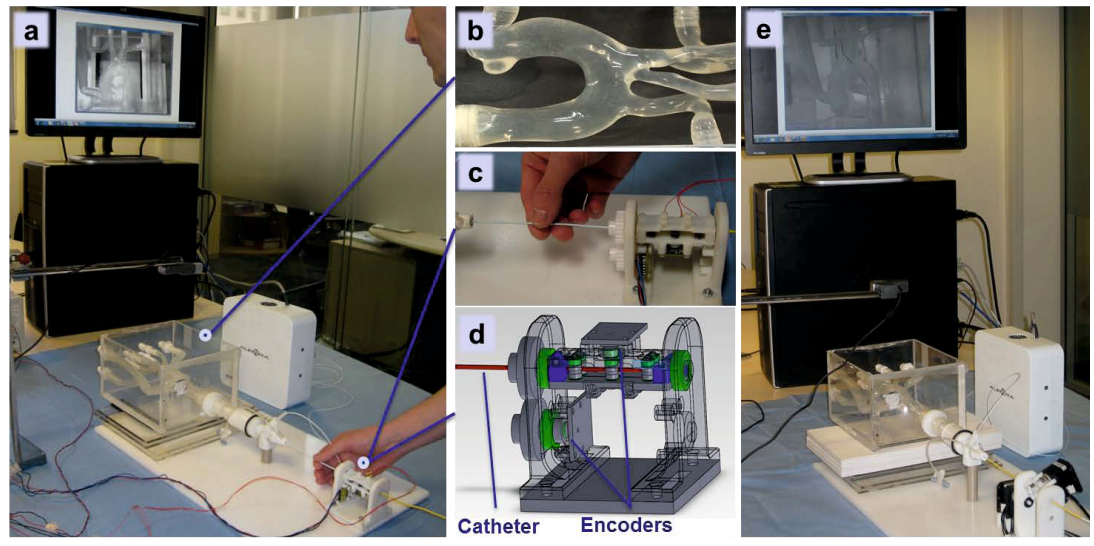

Fig. 1. Experimental setup of measurement unit (a), phantom of the aortic arch (b), proximal position sensor $(\mathrm{c}, \mathrm{d})$, experimental setup of catheter driving unit (e)

into three phases: traversing the descending aorta, moving through the aortic arch, and cannulation of the innominate artery. To improve the quality of data regression, the data at each phase were first temporally aligned using Dynamic Time Warping (DTW). As a result, different training sets consisting of the axial displacement $\left(x_{j}=\left\{x_{t, j}, x_{s, j}\right\}\right)$ and the rotation $\left(\theta_{j}=\left\{\theta_{t, j}, \theta_{s, j}\right\}\right)$ were generated for each of the two experience groups at each phase of the procedure, where the first and second parameters correspond to the temporal and spatial components respectively.

In order to create a probabilistic representation of the temporally aligned phase data that were obtained from the demonstrations, a Gaussian Mixture Model (GMM) was used. By encoding the time signals directly into the model (as an additional dimension) a smooth signal can then be obtained through regression. For a data set represented by $x_{j}$ a GMM of $\mathrm{K}$ components is defined by the following probability density function:

$$
p\left(x_{j}\right)=\sum_{k=1}^{K} p(k) p\left(x_{j} \mid k\right) .
$$

$p\left(x_{j} \mid k\right)=\mathcal{N}\left(x_{j} ; \mu_{k}, \Sigma_{k}\right)$ is the conditional probability density function, $p(k)$ is the prior and $\left\{\mu_{k}, \Sigma_{k}\right\}$ define the mean and covariance matrix of the Gaussian component $\mathrm{k}$ respectively . The Expectation Maximization (EM) algorithm was used to perform maximum-likelihood estimation of the Gaussian mixture parameters, with an initialization obtained through $k$-means clustering [12. The optimal number of components $(K)$ for the GMM model was found using the Bayesian information criterion (BIC), by estimating multiple GMMs with increasing number of components $(K=1$ to 10$)$ and selecting the model with the minimum BIC score. Individual mixture models were created at each phase of the procedure for the axial and rotational motion trajectories. 
To reconstruct smooth trajectories that capture the essential features of the data encoded in the mixture models, Gaussian Mixture Regression (GMR) was implemented by using a sequence of time-steps $\left(x_{t}\right)$ as query points and estimating the expected corresponding spatial distribution $\left(\hat{x}_{s}\right)$ through the regression 12. The temporal and spatial components of the GMM are separated and the conditional expectation $\left(\hat{x}_{s, k}\right)$ and conditional covariance $\left(\hat{\Sigma}_{s, k}\right)$ for each Gaussian $k$, given $x_{t}$, are calculated. These are then combined for a mixture of $\mathrm{K}$ components, based on the probability of the Gaussian component k (2), to calculate the expected spatial distribution and associated covariances $\left\{\hat{x}_{s}, \hat{\Sigma}_{s}\right\}$ at different time steps $x_{t}$.

$$
\beta_{k}=\frac{p\left(x_{t} \mid k\right)}{\sum_{i=1}^{K} p\left(x_{t} \mid i\right)} .
$$

As a result a smooth generalized form of the axial and radial motion trajectories and their associated covariance matrices was produced for each of the experienced and intermediate training sets at each phase of the procedure.

\subsection{Platform for Catheter Motion Replication}

The robotic system designed for validating the generated trajectories (Fig. 1) uses a similar design to the measurement unit with two servomotors (Dynamixel MX-28, RobotIS) that drive the catheter to follow a desired input trajectory (axial and rotational motion) based on a PID controller. The smooth trajectories obtained from the regression are sent to the motors sequentially for each phase of the procedure. At the end of each phase the software interface awaits input from the operator before catheter advancement to the next phase continues.

Cannulation of the innominate artery of the phantom using the robotic driver was performed 12 times with each of the trajectories learned from the two experience levels, across four inexperienced operators. Using the position sensor attached to the tip of the catheter different performance metrics were extracted for each phase of the procedure corresponding to mean and maximum tip velocities, mean and maximum tip acceleration, and number of back and forth movements (corresponding to smoothness of motion). By using these metrics, the performance of the robotic catheterization based on each learned model was compared to the training data obtained from the intermediate and expert operators in the manual setting. Differences between manual and robotic performance were assessed using the non-parametric Wilcoxon rank-sum significance test $(P<0.05)$ on all metrics over each phase of the procedure. Improvements in catheter behaviour between the learned robotic drive and the manual drive are further analyzed by comparing smoothness and overall motion patterns across catheter tip trajectory and displacement plots. All the processing and statistical analysis for this study was performed in Matlab. 


\section{Results}

Fig. 2 shows the mixture model and the trajectory obtained from the regression for the axial motion at each phase of the procedure, as learned from the experienced operator's demonstrations.
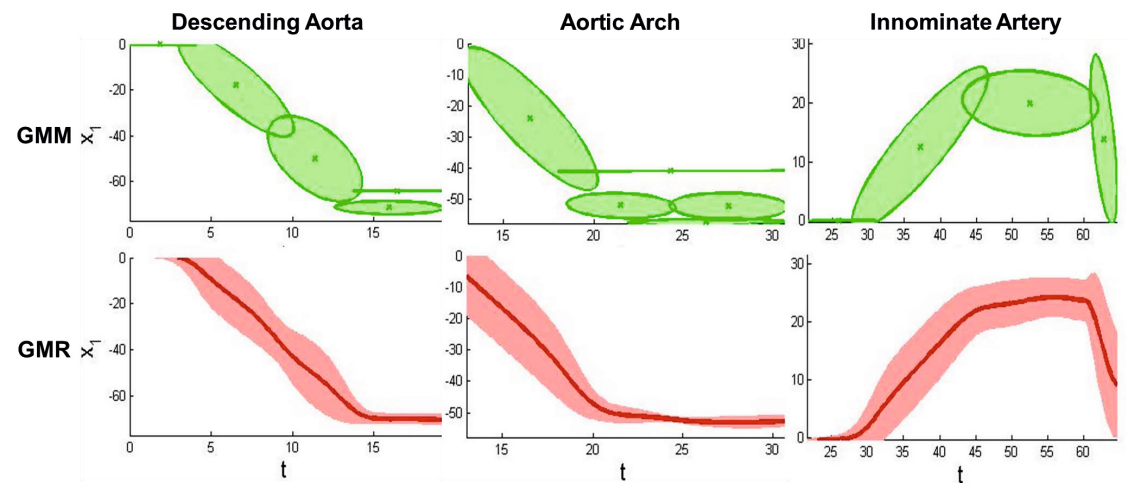

Fig. 2. Plots of GMM (top row) and generated trajectory from GMR (bottom row) for the axial motion learned from the experienced operator for each of the phases of the procedure: descending aorta (left), aortic arch (middle) and innominate artery (right)

Table 1. Median values for statistically significant differences $(P<0.05)$ between robot-assisted learned procedure vs. corresponding manual training data at each phase

\begin{tabular}{lcc|cc|cc}
\hline & \multicolumn{2}{c|}{ Descending aorta } & \multicolumn{2}{c|}{ Aortic arch } & \multicolumn{2}{c}{ Innominate } \\
\hline & \multicolumn{3}{c|}{ Robot Intermediate } & Robot Intermediate & Robot Intermediate \\
\hline mean speed $(\mathrm{mm} / \mathrm{s})$ & 3.3 & 8.7 & 2.9 & 9.1 & 2.5 & 10.2 \\
max speed $(\mathrm{mm} / \mathrm{s})$ & 31.5 & 184.1 & 47.4 & 168.9 & 25.9 & 112.9 \\
mean accel. $\left(\mathrm{mm} / \mathrm{s}^{2}\right)$ & 69.2 & 228.1 & 62.9 & 242.2 & 49.4 & 272.7 \\
max accel. $\left(\mathrm{mm} / \mathrm{s}^{2}\right)$ & $8.7 \mathrm{e} 2$ & $4.8 \mathrm{e} 3$ & $1.5 \mathrm{e} 3$ & $5.4 \mathrm{e} 3$ & $6.0 \mathrm{e} 2$ & $3.3 \mathrm{e} 3$ \\
back/forth movements & 18 & 34 & - & - & - & - \\
\hline & Robot & Expert & Robot & Expert & Robot & Expert \\
\hline mean speed $(\mathrm{mm} / \mathrm{s})$ & 4.0 & 11.6 & 2.9 & 9.4 & - & - \\
max speed $(\mathrm{mm} / \mathrm{s})$ & 54.5 & 213.2 & 41.8 & 81.1 & - & - \\
mean accel. $\left(\mathrm{mm} / \mathrm{s}^{2}\right)$ & 73.1 & 270.1 & 53.1 & 268.7 & - & - \\
max accel. $\left(\mathrm{mm} / \mathrm{s}^{2}\right)$ & $1.4 \mathrm{e} 3$ & $5.5 \mathrm{e} 3$ & $9.8 \mathrm{e} 2$ & $2.7 \mathrm{e} 3$ & - & - \\
back/forth movements & - & - & - & - & 24 & 61 \\
\hline
\end{tabular}

Table 1 shows the result of the non-parametric test, with median values for statistically significant differences between metrics $(P<0.05)$. It is evident that there are significant performance differences between the robot-assisted catheterization based on each learned model and the corresponding manual training set. The results from the model learned from intermediate-skilled operators show smoother and safer catheter motion at lower speeds and accelerations across all 
three phases of the procedure compared to the manual performance. For the expert-based model the results show better performance in terms of acceleration and speed in the first two phases. For the last phase the systems achieves smoother motion with reduced number of movements when passing through the narrow artery, which is the more complex part of the procedure. A reduction in movements could potentially translate into reduced vessel wall contact and therefore less risk of embolization and stroke.

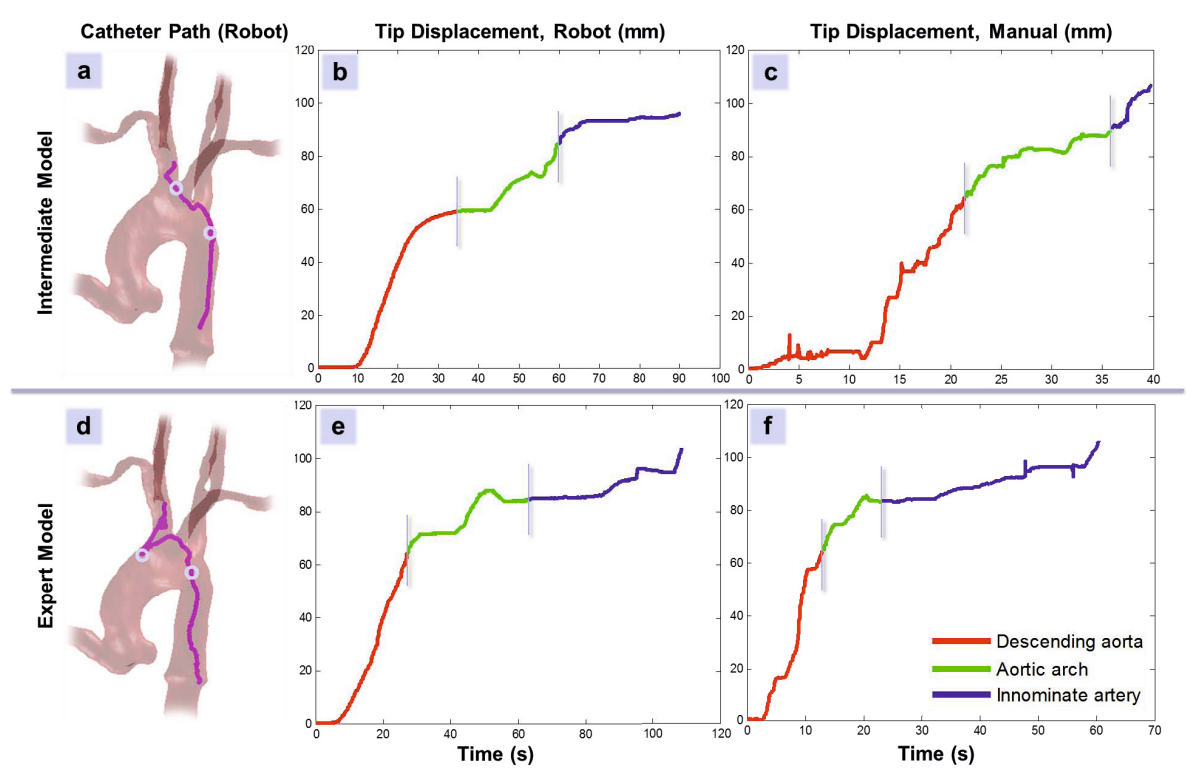

Fig. 3. Catheter tip trajectory and displacement achieved by the robot based on the intermediate model $(\mathrm{a}, \mathrm{b})$ and the expert model $(\mathrm{d}, \mathrm{e})$ and examples of manual catheterization from each of their respective training sets (c) and (f)

Fig. 3 shows the catheter path and tip displacement obtained from the catheter when it was driven by the robot with each of the models from the intermediate group $(\mathrm{a}, \mathrm{b})$ and the expert group (d,e), against an example of their respective training sets (intermediate in (c), expert in (f)). In both cases the results show smoother and more continuous tip motion of the robotic-driven catheter compared to the performance of the training data that was used to generate the proximal motion trajectories, across all three phases of the procedure. The catheter paths $(\mathrm{a}, \mathrm{d})$ also depict the distinct difference in approach between the two experience groups.

\section{Conclusion}

This paper proposes a learning-from-demonstration framework for robot-assisted catheterization, which offers new possibilities for operator-robot collaboration 
and semi-automation of procedures to reduce the cognitive workload of the surgeons. The proposed approach allows for generation of optimum motion trajectories across different phases of an endovascular procedure, as learned from multiple demonstrations of skilled catheterization. Motion models generated from different experience levels are validated using a robotic catheter driver across inexperienced operators, which allows manual control of the guidewire to utilize natural operator skill and ensure procedure safety. The framework can be extended by using the motion primitives for each phase to describe anatomical variations across multiple phantom settings with physiological motion and pulsatile flow. The results depict significant improvements in the quality of catheter navigation, particularly over more complex parts of the anatomy, and provide important insights into the design of more ergonomically sound, hands-on, shared controlled robotic platforms that maintain the natural skills of operators.

Acknowledgments. The authors would like to acknowledge Dr. Celia Riga, Christopher Payne, and Petros Giataganas for their contribution to this work.

\section{References}

1. Riga, C., Bicknell, C., Hamady, M., Cheshire, N.: Evaluation of robotic endovascular catheters for arch vessel cannulation. J. Vasc. Surg. 54(3), 799-809 (2011)

2. Riga, C., Bicknell, C., Rolls, A., Cheshire, N., Hamady, M.: Robot-assisted fenestrated endovascular aneurysm repair (FEVAR) using the Magellan system. J. Vasc. Interv. Radiol. 24(2), 191-196 (2013)

3. Srimathveeravalli, G., Kesavadas, T., Li, X.: Design and fabrication of a robotic mechanism for remote steering and positioning of interventional devices. Int. J. Med. Robot. Comp. 6(2), 160-170 (2010)

4. Payne, C., Rafii-Tari, H., Yang, G.Z.: A force feedback system for endovascular catheterisation. In: IEEE/RSJ International Conference on Intelligent Robots and Systems, pp. 1298-1304 (2012)

5. Rafii-Tari, H., Payne, C.J., Riga, C., Bicknell, C., Lee, S.-L., Yang, G.-Z.: Assessment of navigation cues with proximal force sensing during endovascular catheterization. In: Ayache, N., Delingette, H., Golland, P., Mori, K. (eds.) MICCAI 2012, Part II. LNCS, vol. 7511, pp. 560-567. Springer, Heidelberg (2012)

6. Lin, P., Bush, R., Peden, E., Zhou, W., Guerrero, M., Henao, E., Kougias, P., Mohiuddin, I., Lumsden, A.: Carotid artery stenting with neuroprotection: assessing the learning curve and treatment outcome. Am. J. Surg. 190(6), 855-863 (2005)

7. Hausegger, K.A., Schedlbauer, P., Deutschmann, H.A., Tiesenhausen, K.: Complications in endoluminal repair of abdominal aortic aneurysms. Eur. J. Radiol. 39(1), 22-33 (2001)

8. Thakur, Y., Holdsworth, D.W., Drangova, M.: Characterization of catheter dynamics during percutaneous transluminal catheter procedures. IEEE Trans. Biomed. Eng. 56(8), 2140-2143 (2009)

9. Argall, B.D., Chernova, S., Veloso, M., Browning, B.: A survey of robot learning from demonstration. Robot. Auton. Syst. 57(5), 469-483 (2009) 
10. Reiley, C.E., Plaku, E., Hager, G.D.: Motion generation of robotic surgical tasks: Learning from expert demonstrations. In: Annual International Conference of the IEEE Engineering in Medicine and Biology Society, pp. 967-970 (2010)

11. Padoy, N., Hager, G.: Human-machine collaborative surgery using learned models. In: IEEE International Conference on Robotics and Automation, pp. 5285-5292 (2011)

12. Calinon, S., Guenter, F., Billard, A.: On learning, representing, and generalizing a task in a humanoid robot. IEEE T. Syst. Man. Cy. B. 37(2), 286-298 (2007) 